EPJ Web of Conferences 45, 01081 (2013)

DOI: $10.1051 /$ epjconf/20134501081

(C) Owned by the authors, published by EDP Sciences, 2013

\title{
Numerical investigation of cavitation flow in journal bearing geometry
}

\author{
M. Riedel ${ }^{1}$, M. Schmidt ${ }^{1}$ and P. Stücke ${ }^{1}$ \\ ${ }^{1}$ Faculty of Automotive Engineering, West Saxon University of Applied Science, Zwickau, Germany
}

\begin{abstract}
The appearance of cavitation is still a problem in technical and industrial applications. Especially in automotive internal combustion engines, hydrodynamic journal bearings are used due to their favourable wearing quality and operating characteristics. Cavitation flows inside the bearings reduces the load capacity and leads to a risk of material damages. Therefore an understanding of the complex flow phenomena inside the bearing is necessary for the design development of hydrodynamic journal bearings. Experimental investigations in the fluid domain of the journal bearing are difficult to realize founded by the small dimensions of the bearing. In the recent years more and more the advantages of the computational fluid dynamics (CFD) are used to investigate the detail of the cavitation flows. The analysis in the paper is carried out in a two-step approach. At first an experimental investigation of journal bearing including cavitation is selected from the literature. The complex numerical model validated with the experimental measured data. In a second step, typically design parameters, such as a groove and feed hole, which are necessary to distribute the oil supply across the gap were added into the model. The paper reflects on the influence of the used design parameters and the variation of the additional supply flow rate through the feed hole regarding to cavitation effects in the bearing. Detailed pictures of the three-dimensional flow structures and the cavitation regions inside the flow film of the bearing are presented.
\end{abstract}

\section{Introduction}

The hydrodynamic journal bearing offer a wide range of application. This is due to the simple design and high sustainability. The life of a plain bearing depends on many factors. Among other cavitation can lead to a breakdown of the bearing. Investigation of cavitation in journal bearings was done for more than 50 years.

To detect the possible causes for the breakdown, it is necessary to understand the flow regime inside the bearing. Therefore experiments and numerical simulations were done. Cavitation is founded by three dimensional effects. That's why the investigations were done with the 3D-CFD Code OpenFOAM. The advantage of numerical simulations is that it is possible to see every detail of the flow field. In experiments it is almost impossible to see 3D effects like vortex. On the other hand an experiment is necessary to validate a numerical solution. Especially in the given case the numerical solver for cavitation need to be validated by an experiment. Therefore an experiment was selected from the literature. A numerical model was designed based on the experiment. In the first step the validation is done. Furthermore typical geometrical properties are added to the existing model to see what happen to the cavitation region and pressure curve. The geometrical changes are a groove in circumferentially direction and cross-flow of oil support by a feed hole in radial direction.

\section{Approach}

\subsection{Experimental setup}

The experiment was done by Jakobsson et.al. [1]. The configuration is shown in fig. 1.

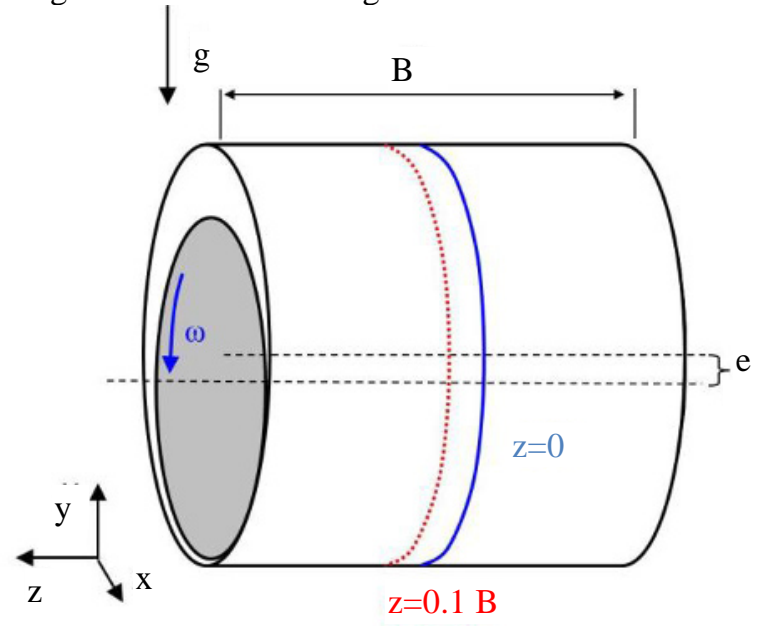

Fig. 1. Experimental setup 
The shaft rotates with the angular velocity $\boldsymbol{\omega}$ and is eccentric to the outer cylinder with the eccentricity e. The gap between inner and outer cylinder is filled with oil. Pressure curve are measured along the line $z=0.1 \mathrm{~B}$. This line is used to compare the computational results with the experimental results. Cavitation is reached downstream the smallest gap. The pressure in that region stays constant. In table 1 the geometrical properties of the experiment are given.

The flow regime inside the system is laminar with a Reynolds number $R e=23$ based on equation 1 .

$$
R e=\frac{\omega R_{1} H_{0}}{v}
$$

Table 1. Properties of the experiment

\begin{tabular}{|c|c|}
\hline Radius of the shaft & $50 \mathrm{~mm}$ \\
\hline $\begin{array}{c}\text { Radius of the } \\
\text { bearing }\end{array}$ & $50.146 \mathrm{~mm}$ \\
\hline $\begin{array}{c}\text { Width of the } \\
\text { bearing }\end{array}$ & $133 \mathrm{~mm}$ \\
\hline Eccentricity & $0.08876 \mathrm{~mm}$ \\
\hline $\begin{array}{c}\text { Angular velocity } \\
\text { Fluid density }\end{array}$ & $840 \mathrm{~kg} \mathrm{~m}^{-3}$ \\
\hline $\begin{array}{c}\text { Kinematic } \\
\text { viscosity of the } \\
\text { fluid }\end{array}$ & $1.51 \cdot 10^{-5} \mathrm{~m}^{2} \mathrm{~s}^{-1}$ \\
\hline $\begin{array}{c}\text { Vapour pressure } \\
\text { of the fluid }\end{array}$ & $20 \mathrm{kPa}^{-1}$ \\
\hline
\end{tabular}

\subsection{Numerical setup}

The simulations are done with the CFD-Code OpenFOAM. The numerical code is based on the finite volume method and solves the three dimensional momentum and continuity equations. OpenFOAM includes a solver for cavitation. These solvers use the volume of fluid-method (VoF) to model the two phase flow. The volume fraction $\alpha$ is calculated by equation 4 . The phase change model base on a bubble dynamic approach according to Sauer [2]. A description about the cavitation solver is given in $\mathrm{Lu}$ [3].

$$
\begin{gathered}
\frac{\partial \rho}{\partial t}+\nabla \rho \mathbf{u}=0 \\
\frac{\partial \mathbf{u}}{\partial t}+(\mathbf{u} \cdot \nabla) \mathbf{u}=\rho g-\frac{1}{\rho} \nabla p-F_{S}+v \Delta \mathbf{u} \\
\frac{\partial \alpha}{\partial t}+\nabla \cdot(\alpha \mathbf{u})=\frac{\dot{m}}{\rho} \\
\alpha=\frac{V_{l}}{V_{l}+V_{v}}
\end{gathered}
$$

The basic model for validation of the numerical solution consists of the fixed outer cylinder and the

rotating inner cylinder. Further design steps include a groove and a feed hole. The ends of the bearing are designed as open ends with ambient pressure boundary condition. The numerical grid is generated as a block structured mesh with hexagonal cells. The feed hole pipe is meshed with a typical O-grid. In radial direction the number of cells is 14 , recommended by Schmidt et.al. [4].

The overall model is shown in fig. 2 and the boundary conditions are listed in table 2. To orientate in circumference direction the angle $\varphi$ is introduced. The widest gap and the feed hole are at the position $\varphi=0^{\circ}$. The positive direction is defined by the convergent gap.

The incoming flow rate is equal to the inner volumetric flow rate calculated by equation 6 .

$$
Q_{0}=\frac{1}{2} B H_{0} U
$$

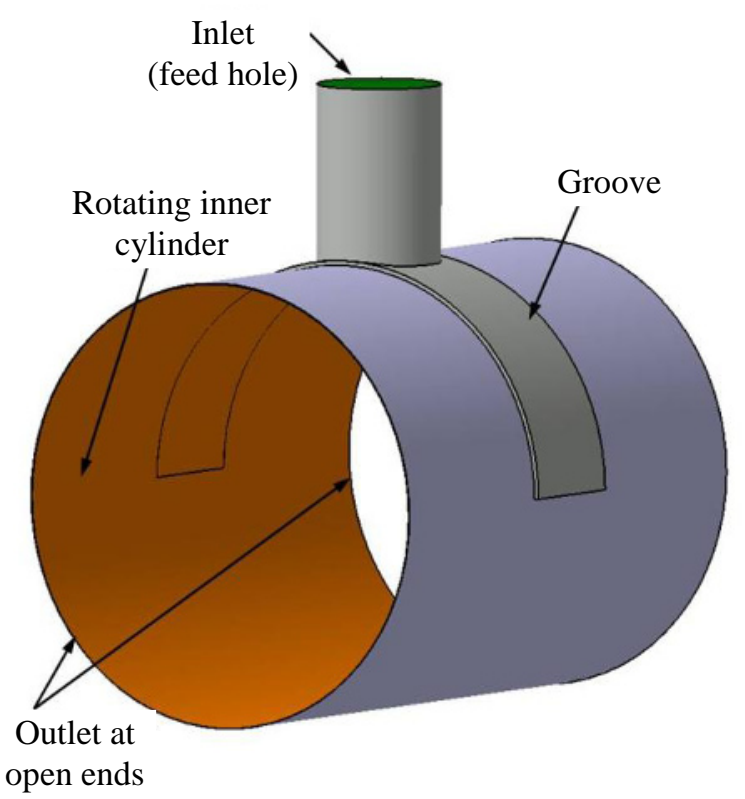

Fig. 2. Boundary at the simulation model

Table 2. Boundary conditions for the simulation.

\begin{tabular}{|l|c|c|c|}
\hline & $\begin{array}{c}\text { Volume } \\
\text { fraction }\end{array}$ & Pressure & Velocity \\
\hline Inner cylinder & $\nabla \alpha=0$ & $\nabla p=0$ & $\boldsymbol{\omega}=48.1 \mathrm{~s}^{-1}$ \\
\hline Inlet & $\alpha=1$ & $\nabla p=0$ & calculated \\
\hline Outlet & $\alpha=1$ & $p=10^{5} P a$ & $\nabla \cdot \mathbf{u}=0$ \\
\hline $\begin{array}{l}\text { Walls (outer } \\
\text { cylinder, groove } \\
\text { and feed hole) }\end{array}$ & $\nabla \alpha=0$ & $\nabla p=0$ & $\mathbf{u}=(0,0,0)$ \\
\hline
\end{tabular}




\section{Results and discussion}

\subsection{Validation of the experiment}

In the first step the numerical simulation validated with the experimental measurement. In fig. 3 the comparison of experiment and simulation is illustrated. It should be observe that there is no groove and no additional flow.

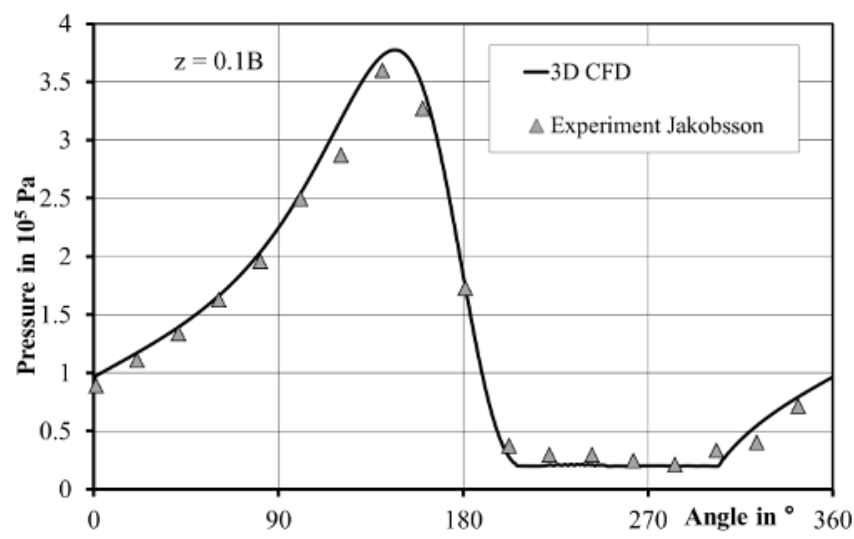

Fig. 3. Pressure plot of experiment and simulation

The simulation is in good agreement to the experiment. In the divergent gap the pressure reaches the value of the fluid vapour pressure hence in fluid vaporization. In fig. 4 the isosurface of the volume fraction for vapour $(\alpha<0.95)$ is shown. It is obvious that the vapour region reaches the largest degree in the symmetry plane of the bearing. Both, the divergent geometry of the gap and the ambient pressure at the ends influence the distribution of the vapour region. Therefore the vapour volume fraction decreases to the outlets.

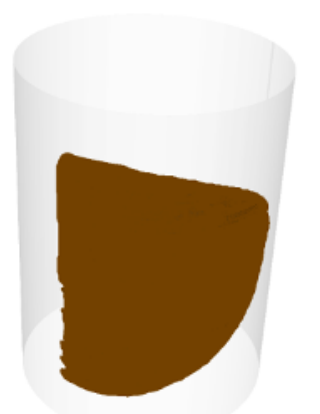

Fig. 4. Vapour region downstream the smallest gap

\subsection{Without groove and with cross-flow}

In a second step some geometrical changes are done. First a feed hole is added to simulate fluid support by crossflow. The comparison of the pressure curves is illustrated in fig. 5. Caused by the volumetric flow the maximum pressure level is higher than in the experiment. The influence of the cross-flow to the pressure is obvious in the region $\varphi= \pm 20^{\circ}$. The incoming fluid affects the pressure in that region (shown by the fluctuation there). The size of the cavitation region is also affected by the higher pressure level in general. The shape of the cavitation region is similar to the simulation without cross-flow. The size of the cavitation region is smaller than in the simulation of the experiment. The reason for that is the higher pressure level in general.

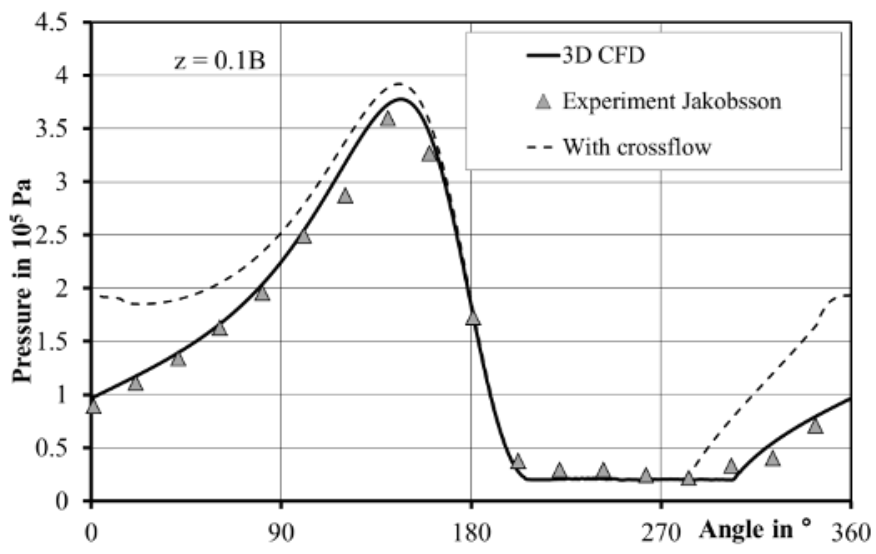

Fig. 5. Pressure plot of experiment, simulation of experiment and simulation with cross-flow

\subsection{With groove and without cross-flow}

In the next step the model extended with a groove. The groove improves the oil supply for the journal bearing. The groove consider $\varphi= \pm 90^{\circ}$ around the widest gap. Fig. 6 shows the comparison of the pressure curve between the experiment and the simulation with groove. It is obvious that the groove has an influence on the pressure curve and cavitation region. The maximum pressure is $13.2 \%$ less than without groove. Founded in the larger gap size caused by the groove the pressure generation is affected. In the groove the pressure is nearly on a constant level. The reason for that is the wider gap there. This is also a reason for a smaller cavitation region. The shape of the cavitation region is influenced by the groove fig. 7 . In the middle of the cylinder it is a bit compressed, founded in the position of the groove. The whole cavitation region is smaller than without groove.

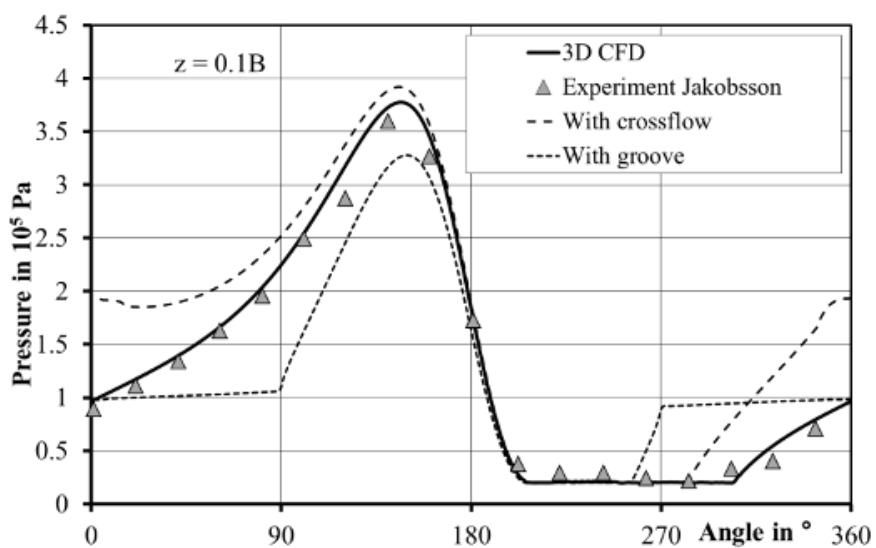

Fig. 6. Pressure plot of experiment, simulation of experiment, simulation with cross-flow and simulation with groove 


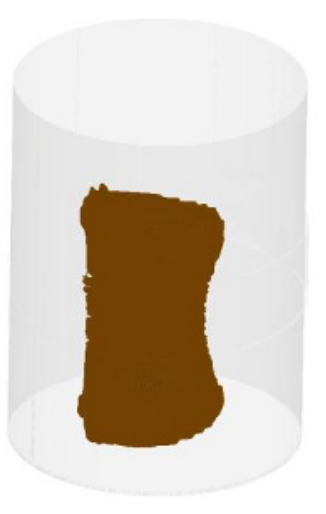

Fig. 7. Vapour region, model with groove

\subsection{With groove and with cross-flow}

Typical for journal bearing is a configuration with groove and cross-flow. In fig. 8 the pressure curve is shown. The maximum pressure is higher than without cross-flow but smaller than without groove. The pressure gradient in the whole region is similar to the gradient of the model with groove and without cross-flow.

The Cavitation region is decreasing caused by the higher pressure level in the groove. The shape of the cavitation region is similar to fig. 7 but, like mentioned before, there is less vapour volume fraction.

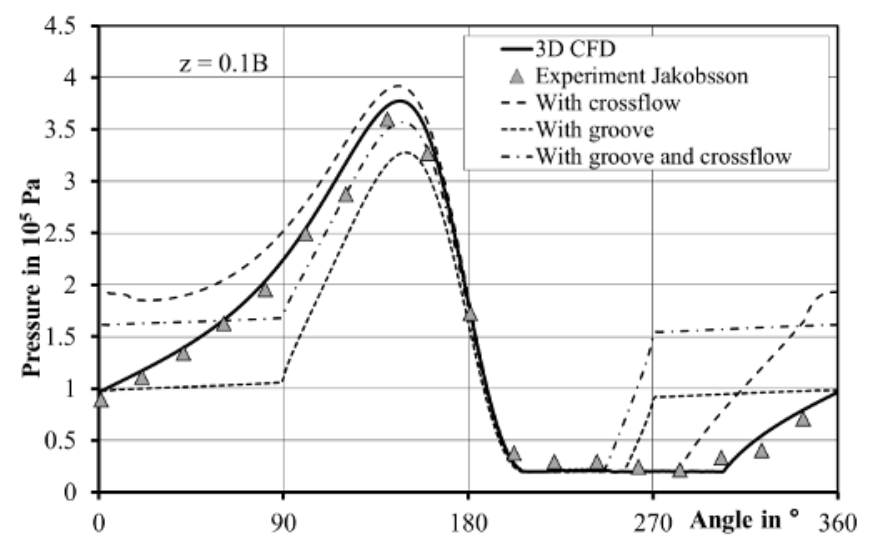

Fig. 8. Pressure plot of the experiment and all simulations

\section{Conclusions}

In this paper the solver for cavitation could validate experimental results from the literature. Based on the simulation model some changes in the geometry were done. These changes give influence to the size and shape of the cavitation region. The changes in the pressure plot could be explained and appeared logical. With this knowledge calculations in journal bearing considering cavitation effects can be performed.

In real journal bearing the rate of cross flow varies. The volumetric flow depends on the flow rate from the oil pump. Therefore calculations with variation of the incoming volumetric flow have to be done. Furthermore the displacement of the shaft should be considered. The shaft moves on a trajectory hence the eccentricity is also moving. It is also necessary to observe this circumstance.

\section{Nomenclature}

$\begin{array}{ll}B & \text { Breadth of the bearing } \\ e & \text { Eccentricity } \\ F_{S} & \text { Surface tension force } \\ g & \text { Gravity acceleration } \\ H_{0} & \text { Bearing clearance } \\ \dot{m} & \text { Mass flow rate } \\ Q_{0} & \text { Volumetric flow } \\ p & \text { Pressure } \\ R_{1}, R_{2} & \text { Radius of shaft and outer cylinder } \\ R e & \text { Reynolds number } \\ t & \text { Time } \\ \mathbf{u} & \text { Velocity vector } \\ U & \text { Velocity in circumference direction } \\ V_{l}, V_{v} & \text { Volume of liquid and vapour } \\ x, y, z & \text { Cartesian coordinates } \\ \alpha & \text { Volume fraction } \\ v & \text { Kinematic viscosity } \\ \rho & \text { Density } \\ \boldsymbol{\omega} & \text { Angular velocity }\end{array}$

\section{References}

1. B. Jakobsson, L. Floberg.: The finite journal bearing considering vaporization. Chalmers Tekniska Högskolas Handlingar (1957)

2. J. Sauer: Instationär kavitierende Strömungen-Ein neues Modell basierend auf Front Capturing (VoF) und Blasendynamik. University Karlsruhe. Dissertation (2000)

3. N.X. Lu: Solve Cavitating flow around a $2 D$ hydrofoil using a user modified version of interPhaseChangeFoam. Chalmers University of Technology (2008)

4. M. Schmidt, P. Stücke, M. Nobis : Numerical meshing issue for three-dimensional flow simulation in journal bearings. 3rd Micro and Nanoflow Conference in Thessaloniki/GR (2011) 